\title{
I+D+i en educación médica
}

José A. Gutiérrez Fuentes

Sir William Osler, en su discurso de apertura del Instituto Wistar de Anatomía y Biología (Filadelfia, 21 de mayo de 1894), significativamente titulado 'La levadura de la Ciencia', dice: 'Especialmente para el médico, una disciplina científica es un don incalculable que fermenta toda su vida'. Y añade, con mayor énfasis: 'la perdición espera inevitablemente a la mente del médico que nunca ha sido plenamente inoculado con la levadura, que nunca ha captado las relaciones de la ciencia con su arte, y que no sabe nada, y quizá ni le importa, de sus limitaciones'. Así que existe una 'gran necesidad de hombres que sean pensadores además de trabajadores, hombres con ideas, que hayan bebido hasta el fondo la copa del vino astral.... Pero no sólo los conocimientos teóricos -el vino astral- permiten hacer medicina, sino sobre todo ejercitar la mente del médico en la búsqueda de nuevos conocimientos, dado que no existe una separación formal entre la forma mental de hacer medicina y de hacer investigación. Osler insiste mucho en este punto: 'la levadura de la ciencia proporciona a los hombres hábitos de precisión mental. Fortalece -para usar una expresión de Epicarno- los músculos y tendones del entendimiento' [1].

Sin lugar a dudas, la medicina se estructura en torno a la aplicación de conocimientos científicos, datos fácticos en permanente revisión, obtenidos por medio de un proceso de experimentación sistematizado. Sin embargo, y desde el momento que se trata de un conocimiento que el hombre posee y aplica sobre sus pares, la medicina asistencial trasciende la aplicación de información científica.

Surge, en consecuencia, una dimensión interpersonal desde donde se forja la atmósfera tera- péutica, ingrediente tan importante para el logro de una medicina efectiva como la presencia misma en el médico de un conocimiento científico adecuado. Es precisamente en la gestación de dicha atmósfera donde reside el componente personal, casi artístico, del acto médico.

Hace unos años, en un editorial del British Medical Journal, podía leerse que las tres palabras más importantes en educación son 'I don't know' ('no lo sé). Está claro que la práctica médica no es sencilla por su 'inherente incertidumbre', lo que hace que el razonamiento clínico no sea siempre fácil. Por esta razón, no es malo no saber algo siempre que esto nos motive a aprender más y a ayudar a otros a aprender [2].

La educación médica tradicional ha enfocado su énfasis en el desarrollo del conocimiento, las destrezas y actitudes, cuando en el mundo moderno no sólo se debe educar para la competitividad, sino para la capacidad de adaptarse al cambio, de generar nuevo conocimiento y de mejorar continuamente nuestro desempeño. Es en este contexto que la educación médica debe tener muy bien definidas sus metas y procesos para lograr hacer de los estudiantes personas con la capacidad de practicar la medicina. Se trata de lograr que el aprendizaje no sea sólo una transmisión de hechos y contenidos, sino más bien un proceso activo a través del cual a la vez se desarrollen destrezas y sensibilidades para toda la vida.

La medicina científica trata de comprender la razón de los cambios, atribuibles a la enfermedad, acaecidos en la fisiología de un ser biológico particular, el hombre, y de prevenirlos o curarlos, enmarcándose claramente entre las ciencias de la vida, una parte de la biología [3]. Contribuir al progreso de la ciencia médica debe formar parte
Director de la

Fundación Lilly.

E-mail

j_a_gutierrez@lilly.com 
del quehacer de cada médico, para lo que resultan imprescindibles los conocimientos teóricos. La medicina tiene la responsabilidad de investigar para progresar, y si los médicos renunciasen a esa actividad (por ejemplo, desdeñando la adquisición de conocimientos teóricos), la medicina dejaría de ser una ciencia, convirtiéndose en un conjunto acartonado de reglas de actuación [4].

El método clínico es un proceso científico que se desarrolla a partir de que el médico indaga qué le sucede al paciente, lo explora en busca de signos o síntomas orientadores, establece hipótesis diagnósticas que den explicación a lo observado, comprueba las hipótesis generadas a través de las pruebas diagnósticas complementarias, establece los principios causales y adopta la decisión de intervención y el tipo de ésta, a la vez que establece el dictamen de pronóstico causal y evolutivo. En definitiva, estamos ante un planteamiento de investigación que podrá limitarse a lo individual o extrapolarse a lo colectivo. Se trata de la aplicación de conocimientos teóricos (explicativos), a través de un procedimiento científico, para sistematizar la mera observación [3].

Siendo así que la medicina es una disciplina científica, durante el siglo XIX se crearon los primeros laboratorios de investigación en los hospitales y centros asistenciales de las ciudades universitarias más conocidas de algunos países europeos y de Estados Unidos. En éstos han trabajado quienes describieron los principales agentes etiopatogénicos y los mecanismos o parámetros biológicos que subyacen al desarrollo y evolución de las enfermedades.

La cultura de la investigación en los centros sanitarios es esencial y se ha ido fortaleciendo con el paso del tiempo. Razones para ello no faltan: capacita a los profesionales para actuar con criterios científicos ante los pacientes y sus padecimientos, genera colaboraciones tecnológicas externas e intercambios, exige una actualización continua de conocimientos y favorece la incorporación racional de innovaciones científicas y tecnológicas. De ésta surge la formación del médico investigador: es decir, el médico que ha aprendido a investigar y que ayuda a que el sistema sanitario pueda progresar a través de la adquisición de conocimiento. El médico investigador mejora la capacidad de comprender los problemas del paciente y de buscar las alternativas terapéuticas adecuadas, lo que redunda en una mejor calidad asistencial [5].
Las facultades de medicina deberían reconocer como parte fundamental de su misión educativa el promover la investigación y la formación de investigadores. La formación investigadora es una competencia que tendría que iniciarse en la universidad. Sin embargo, hoy día, los contenidos de la carrera no dejan mucho margen para estudiar materias alejadas de la práctica médica, como son la metodología de investigación o los métodos de experimentación médica más actuales, incluida la manipulación del genoma.

Hace unos años, el Howard Hughes Medical Institute, a través de la llamada 'Comisión Boyer', se planteaba los desafíos de la educación científica para estudiantes de pregrado en universidades que realizan investigación en Estados Unidos, y llegó, entre otras, a las siguientes conclusiones [6]:

- Los estudiantes de pregrado que toman clases de ciencia en universidades que realizan investigación se encuentran a menudo en grandes salas de conferencias. La interacción que tienen con científicos investigadores prominentes va de limitada a inexistente.

- La encuesta nacional de enrolamiento de estudiantes del 2001 mostró que sólo el 25\% de los estudiantes del último año de todas las especializaciones en las universidades que otorgan doctorados, trabajaba en investigación con profesores, fuera de los requisitos de los cursos.

- Según el Consejo Nacional de Investigación, muchos estudiantes de pregrado no toman más de un año de ciencia, y la comprensión pública de cómo se hace la ciencia y cómo piensan los científicos es pobre.

- La cultura de las universidades que realizan investigación tiende a infravalorar la enseñanza entre los investigadores.

- Los estudiantes de doctorado y los becarios posdoctorales en ciencias no están entrenados o motivados para la enseñanza.

- Las decisiones sobre titularidad y promociones de los investigadores tienden a basarse casi enteramente en la investigación y las publicaciones, infravalorando la enseñanza.

- Las reuniones científicas nacionales raramente incluyen sesiones que se ocupen de la enseñanza. Cuando lo hacen, esas sesiones no se integran en su totalidad con la reunión, reforzando la separación entre las dos actividades. 


\section{Tabla I. Aspectos negativos.}

Enfoque teórico excesivo

Necesidad de adaptar programas teóricos

Algunas carencias formativas específicas:

relaciones y comunicación

Escaso fomento de la investigación

Examen MIR como referente

Escisión docencia/asistencia

Resistencia al cambio
Por su parte, la creación del Espacio Europeo de Educación Superior (EEES), que tiene su origen en la Declaración de Bolonia (19 de junio de 1999), contempla entre sus objetivos los siguientes:

- Reforzar la competitividad de la educación europea superior.

- Favorecer la tasa de empleo y movilidad de los universitarios.

- Adoptar un sistema comprensible y comparable de estudios.

- Establecer un sistema de créditos como unidad de medida de carga docente: European Credit Transfer System (ECTS).

- Promocionar la movilidad y el intercambio de estudiantes, profesores y profesionales posgraduados entre los países.

En las reuniones de Praga (2001) y Berlín (2003), a estos objetivos se añadieron los tres siguientes:

- Integrar el aprendizaje de por vida (life-long learning) en la estrategia educativa global.

- Implicar a los estudiantes en el desarrollo del proceso.

- Promover la creación de un área europea en investigación.

En España, estas directrices se plasman a través de la orden ministerial (Orden ECI/332/2008, de 13 de febrero), por la que se establecen los requisitos para la verificación de los títulos universitarios oficiales que habiliten para el ejercicio de la profesión de médico.

En el anexo de esta orden se detalla la estructura del nuevo grado de Medicina, siguiendo las
Tabla II. Aspectos positivos.

Alumnos con elevada preparación

Alumnos con alto rendimiento académico

Programas teóricos completos

Inicio de cambios e innovación educativa

Orientación a la adquisición de competencias

clínicas (definición, entrenamiento, evaluación)

directrices del Libro Blanco de ANECA, que determina unos objetivos resumidos en 37 'competencias específicas' más la estructuración del plan de estudios con cinco módulos:

- Morfología, estructura y función del cuerpo humano (64 ECTS).

- Medicina social, habilidades de comunicación e iniciación a la investigación (30 ECTS).

- Formación clínica humana (100 ECTS).

- Procedimientos diagnósticos y terapéuticos (40 ECTS).

- Prácticas tuteladas y trabajo de fin de grado (60 ECTS).

Se reservan los restantes 30 créditos para la oferta de materias optativas.

De todo lo expuesto se deduce una clara prioridad global por el impulso a la I+D, y más concretamente por la investigación en el campo de la medicina. Para poder dar una respuesta adecuada queda patente la necesidad de que nuevos investigadores bien formados y con talento nutran los hospitales y los laboratorios. Este requisito sólo podrá verse satisfecho si se inicia, alienta y forma a los actuales estudiantes en los diferentes campos de la investigación médica.

Recientemente, desde la Cátedra de Educación Médica Fundación Lilly-UCM [7] realizamos una encuesta a los médicos que ejercen docencia de práctica clínica en los tres hospitales vinculados a la Universidad Complutense de Madrid. Se trata de un estudio sociológico, entre los formadores clínicos de las nuevas generaciones de médicos, sobre el actual sistema de formación, las carencias, deficiencias o disfuncio- 
nes que perciben en él, y sus posibles propuestas o sugerencias para mejorarlo. Se efectuaron 435 entrevistas con afijación no proporcional a la distribución del universo entre catedráticos, profesores titulares, profesores asociados, médicos no docentes y médicos residentes. Los resultados de la encuesta pueden verse en las tablas I y II.

Como barreras para la implantación de nuevos modelos, los encuestados identificaron la escasa motivación del profesorado, la falta de reconocimiento docente adecuado y la dedicación temporal.

En resumen, se dan las circunstancias y el aparente convencimiento de que la $\mathrm{I}+\mathrm{D}$ sigue siendo una asignatura pendiente en España. Para salvar esta situación, además de un decidido apoyo político y financiero, es necesario introducir en los planes educativos la información y entrenamiento necesarios para que los estudiantes puedan conocer y valorar la investigación como una parte del ser médico, y los que así lo deseen, hacer de ello su actividad principal. Es precisa la reestructuración de la formación de especialistas en los hospitales, incrementando los contenidos en in- vestigación básica, clínica y epidemiológica, así como en el manejo de instrumentación médica.

\section{Bibliografía}

1. Osler W, ed. Un estilo de vida y otros discursos con comentarios y anotaciones. Madrid: Fundación LillyUnión Editorial; 2007.

2. BMJ Editor's Choice. 'I don't know': the three most important words in education. BMJ 1999; 318: 7193.

3. Mayr E. This is biology, the science of the living world. Harvard: Harvard University Press; 1997.

4. Baquero F. De la enseñanza de los conocimientos teóricos en Medicina. Educ Med 2008; 11 (Supl 1): S17-20.

5. Vidal-Vanaclocha F. La formación investigadora de nuestros médicos: historia y presente. Origen asistencial de la investigación médica. Educ Med 2009; 12 (Supl 3): S51-3.

6. Howard Hughes Medical Institute. Educación científica para estudiantes de pregrado en universidades que realizan investigación. HHMI, 18 de septiembre de 2002.

7. Fundación Lilly. URL: http://www.fundacionlilly.com/ Nitro/foundation/templates/lines.jsp?page $=10007$. 